\title{
Predictive Significance of Renal Histopathology as Correlated with Renal Function in Patients of Nephrotic Syndrome
}

\author{
Sufian Zaheer ${ }^{1 *}$, Preeti Sharma ${ }^{2}$, Mehar Aziz ${ }^{3}$ and Shahzad F Haque ${ }^{3}$ \\ ${ }^{1}$ Professor, Department of Pathology, VMMC and SJH, New Delhi \\ ${ }^{2}$ Senior Resident, Department of Pathology, VMMC and SJH, New Delhi \\ ${ }^{3}$ Professor, Department of Pathology, JNMC, AMU, Aligarh
}

\section{ABSTRACT}

Background: The advent of renal biopsy in early 1950's has greatly enhanced the understanding of renal disease including nephrotic syndrome in all areas of clinical nephrology, pathology and investigations. The biopsy data, complemented by appropriate clinical, laboratory information and basic studies has contributed significantly to the body of knowledge of renal disease.

Material and Methods: This study was undertaken to analyze the usefulness of renal biopsy in patients of nephrotic syndrome. The present study was conducted on 52 patients of nephrotic syndrome, with detailed clinical examinations, relevant biochemical investigations and these patients were subjected to renal biopsy for detailed histopathological examination.

Results: Of the 52 cases, 34 (65.4\%) were male and 18 (34.6\%) were females with male to female ratio of 1.9:1 and mean age being 29.5+ 12.5 years. Pre-biopsy clinical assessmentrevealed the major cause of nephrotic syndrome as primary glomerular disease in 42 (80.8\%). Secondary cause of nephrotic syndrome was suspected in the remaining 10 (19.2\%) of patients. After pathological evaluation, the major cause of nephrotic syndrome observed in the present study was primary glomerular diseases which accounted for 40 (76.9\%) patients. Secondary glomerular diseases were observed in the remaining $12(23.1 \%)$ patients. Post biopsy histopathological diagnosis lead to change in therapy in $67.4 \%$ of the patients and the alteration of therapy post biopsy most commonly revolved around the use of corticosteroid. In 8 patients therapy with cytotoxic drugs was started.

Conclusion: The estimate of prognosis was in agreement (pre-biopsy and post-biopsy) in $61.5 \%$ of cases whereas in 20 (38.5\%) patients it was different. In $12(23.1 \%)$ cases a better prognosis was estimated and in $8(15.4 \%)$ cases it was worse than estimated previously.

Keywords: Nephrotic Syndrome; Renal Biopsy; Primary Glomerular Disease; Secondary Glomerular Disease

\section{Introduction}

Nephrotic syndrome (NS) is the term widely used to describe a clinical situation in which there is heavy loss of protein in the urine, a fall in certain serum protein particularly albumin and the presence of edema. In addition, other biochemical and metabolic abnormalities are found chiefly hypercholesterolemia and the presence of fat bodies in the urine. ${ }^{1}$ Renal biopsy has advanced the understanding of pathological process, the planning of rational therapeutic approaches and has provided a general estimation of prognosis of patient with renal disease. 2,3 However, renal biopsy is costly because of need for both complex laboratory preparation and experienced pathologists who interpret the tissue. A kidney biopsy may be obtained to help establish diagnosis, to direct therapy, or to suggest prognosis. The information from biopsy is still largely qualitative, although morphometric techniques have been developed to quantify histopathologic alterations. These techniques have been used almost exclusively in research, even in these setting there are few data comparing the reproducibility of different techniques to quantify kidney biopsy results. ${ }^{4}$

Clinical investigations can only be arbitrary and incomplete but nevertheless they can be a useful conjunct to renal biopsy interpretation. Renal biopsy also provides valuable and completely essential information regarding the underlying diseases, which can be missed in management of nephrotic syndrome, by routine clinical management. ${ }^{5}$

In the pediatric age group early diagnosis at the onset of renal diseases can only be provided by renal biopsy, which can lead to early effective management and even avert fatal consequences.

The predictive outcome of renal biopsy is the best as compared to any other organ, because of the ever changing panorama of immune complex diseases of the kidney and their possible management and treatment in various phases of the disease. Here we have studied the usefulness of renal biopsy in patients of nephrotic syndrome with regard to diagnosis, therapeutic advantage and estimate of prognosis. 


\section{Material and Methods}

The present study was done on 52 cases of adult nephrotic syndrome that were admitted to medical wards and attended renal clinics. The patients, presenting with the features of nephrotic syndrome in whom a successful percutaneous renal biopsy could be performed, were selected for the study. A detailed clinical history was taken and a thorough clinical examination was done. In addition to this, the patients were investigated with haemogram including general blood picture, sputum for ZN staining and culture, blood sugar, renal function test, lipid profile, serum protein including total serum protein (TSP) and A/G ratio, bleeding time and clotting time, urine analysis, 24 hours urinary protein examination, urine culture, X -ray chest, PA View, and USG Abdomen including kidney, ureter and bladder.

The patients of nephrotic syndrome underwent biopsy either under direct ultrasonographic guidance, or the renal size, depth, lower pole localization and cortical thickness was predetermined and the position marked on the patient's back. The kidney biopsy was performed by disposable semi-automatic spring loaded mechanism renal biopsy gun (Bard). The biopsy material obtained was kept immediately in 10\% formalin in a container and sent for histopathological examination incorporating Hematoxylin and Eosin (H\& E), Periodic Acid Schiff (PAS), Reticulin, Van Geison and Methylene blue stains. The present study evaluated the different types of primary and secondary glomerular disease on specific histopathological classification. The histopathological picture was correlated with various clinical and biochemical parameters in predicting the therapeutic outcome and prognostic outcome.

\section{Results}

Fifty two patients of nephrotic syndrome, admitted to the medical wards and attending renal clinic were evaluated. The mean age of patients in this study was $29.5+12.5$ years out of which $34(65.4 \%)$ were males and 18 (34.6\%) were females. The duration of the disease in 6 cases was less than a month, 22 cases were having the disease for more than a month to up to one year, 9 cases had the disease for a period ranging from 1 to 2 years, 6 cases had the disease for 2-3 years, 4 cases for 3-4 years and, 3 cases for a period of 4-5 years and the remaining 2 cases had the disease for $>5$ years.

The patients presented with the clinical features as shown in table I. Generalized edema was present in all patients of each group as this is one of the diagnostic criteria of nephrotic syndrome. Pallor was also a common finding seen in overall 36 patients. History of fever was present in a total 16 patients. Eight out of the 11 patients of renal amyloidosis, 3 out of 6 patients of acute diffuse proliferative (post-infectious) glomerulonephritis, 2 patients of focal segmental glomerulonephritis (FSGS) and one patient each of minimal change disease (MCD), membranous glomerulonephritis (MGN) and membranoproliferative glomerulonephritis (MPGN) were febrile. Loss of appetite was present in 7 out of 11 patients of renal amyloidosis, both the two patients of chronic glomerulonephritis and 1 case of membranous glomerulonephritis. History of cough with expectoration was present in 8 patients of renal amyloidosis and in one patient of membranoproliferative glomerulonephritis. Seventeen out of the total 52 patients in our study were hypertensive, including 4 patients of acute diffuse proliferative (post-infectious) glomerulonephritis, 3 patients each of MPGN and renal amyloidosis. Both the 2 patients of chronic glomerulonephritis and 2 out of the 6 cases of membranous glomerulonephritis, and 1 patient each of FSGS, mesangioproliferative glomerulonephritis and a single patient of diabetic nephropathy was hypertensive.

Overall 17 patients had microscopic haematuria on urine examination, which was present in 4 patients of acute diffuse proliferative (post-infectious) glomerulonephritis and both the two patients of chronic glomerulonephritis. Microscopic haematuria was also present in 4 out of the 12 patients of MPGN, 2 patients each of FSGS, MGN and mesangioproliferative glomerulonephritis, and in 1 out of 11 patients of renal amyloidosis. Microscopic haematuria was not observed in any patient of MCD nor was it seen in a patient of diabetic nephropathy. In 2 patients macroscopic haematuria was present, both the patients were of acute diffuse proliferative (post-infectious) glomerulonephritis. Of the total patients, 15 were oliguric and had features of renal dysfunction. 3 of the 11 patients of renal amyloidosis, both the 2 patients of chronic glomerulonephritis and 2 patients each of MGN, acute proliferative (post-infectious) glomerulonephritis and MPGN were oliguric and had renal dysfunction. Oliguria and features of renal dysfunction was also present in 1 patient each of minimal change disease, FSGS and mesangioproliferative glomerulonephritis, and in the patient of diabetic nephropathy.

Table II shows distribution of proteinuria/ $24 \mathrm{hrs}$. The value of total serum protein (TSP) ranged from 2.8 to $5.9 \mathrm{gms} /$ $\mathrm{dl}$, with a mean value of $4.23 \pm 0.94 \mathrm{gms} / \mathrm{dl}$, while the mean value of S. albumin was $1.90 \pm 0.44 \mathrm{gms} / \mathrm{dl}$ ranging from 1.0 to $2.6 \mathrm{gm} / \mathrm{dl}$. The serum globulin level ranged from $1.3-$ $3.4 \mathrm{gms} / \mathrm{dl}$ with the mean value of $2.36 \pm 0.54 \mathrm{gms} / \mathrm{dl}$. The overall mean of total serum protein and serum albumin was decreased, and the overall mean of serum globulin was at the lower side of normal range.

Table III shows the mean value and range of lipid profile in the patients studied. The mean value of all the constituents 
of the lipid profile was raised except the mean value of high density lipoproteins (HDL), which was within the normal range. The mean value of blood urea was $48.54 \pm 11.81 \mathrm{mg} /$ $\mathrm{dl}$, with the values ranging between $32-88 \mathrm{mg} / \mathrm{dl}$ and the value of serum creatinine ranged from 0.8 to $5.1 \mathrm{mg} / \mathrm{dl}$ with a mean value of $1.59+0.81 \mathrm{mg} / \mathrm{dl}$

Provisional clinical (pre-biopsy) diagnosis was made on the basis of clinical history and relevant investigations in patients of nephrotic syndrome. In 42 patients, the diagnosis of primary glomerular disease was made after ruling out secondary causes of nephrotic syndrome. In 10 patients $(19.2 \%)$ secondary cause of nephrotic syndrome was suspected, in the form of chronic systemic disease with deranged renal function in 9 patients and diabetic nephropathy in 1 patient.

According to the various histopathological features noted on $\mathrm{H} \& \mathrm{E}$ staining as well as on special staining the diagnosis of primary glomerular disease was made in 40 (76.9\%) patients and that of secondary glomerular disease in $12(23.1 \%)$ cases. Diagnosis of MCD and FSGS was made in $5(9.6 \%)$ cases each, $6(11.5 \%)$ patients each were diagnosed with membranous glomerulonephritis and acute diffuse proliferative (post-infectious) glomerulonephritis. The histopathological diagnosis of MPGN was made in $12(23.1 \%)$ patients while that of mesangioproliferative glomerulonephritis was made in 4 (7.7 \%) patients.
Further two $(3.9 \%)$ patients showed features of chronic glomerulonephritis in which no secondary associated cause was present. Of the patients diagnosed with secondary glomerular disease after histopathological examination, diagnosis of renal amyloidosis was made in $11(21.2 \%)$ patients. Out of this six patients came out to be cases of pulmonary tuberculosis after thorough clinical examination and investigations including chest X-ray and sputum examination. Further two patients had prior history of pulmonary tuberculosis with inadequate duration of anti-tubercular therapy, 2 patients were suffering from chronic obstructive pulmonary disease (COPD), and in 1 case no cause was obtained on thorough examination. The remaining $1(1.9 \%)$ patient revealed diabetic nephropathy.

Estimate of prognosis (pre-biopsy vs. post-biopsy): The clinical estimate of prognosis was made by assessing age, duration of disease, blood pressure, total urinary protein and volume, renal function tests, serum proteins, lipid profile and steroid responsiveness. The histopathological assessment of prognosis was done by information provided by renal biopsy-glomerular crescent, segmental glomerular sclerosis, and degree of tubular atrophy, interstitial fibrosis and vascular changes. The estimate of prognosis was in agreement (pre-biopsy and post-biopsy) in $61.5 \%$ of cases whereas in $20(38.5 \%)$ patients it was different. In 12 $(23.1 \%)$ cases a better prognosis was estimated and in 8 $(15.4 \%)$ cases it was worse than estimated previously.

Table I: Clinical feature in cases studied.

\begin{tabular}{|l|c|c|}
\hline \multirow{2}{*}{ Clinical features } & \multicolumn{2}{|c|}{ Cases (n = 52) } \\
\cline { 2 - 3 } & No. of cases & Percentage \\
\hline Anasarca & 52 & 69.2 \\
\hline Pallor & 36 & 30.8 \\
\hline Fever & 16 & 100 \\
\hline Loss of appetite & 10 & 17.3 \\
\hline Cough with expectoration & 9 & 32.7 \\
\hline Hypertension & 17 & 32.7 \\
\hline Microscopic Haematuria & 17 & 3.8 \\
\hline Macroscopic haematuria & 2 & 28.8 \\
\hline Oliguria & 15 & 28.8 \\
\hline Renal dysfunction & 15 & \\
\hline
\end{tabular}

Table II: Degree of proteinuria in patients studied

\begin{tabular}{|c|c|c|c|}
\hline S. No. & $\begin{array}{c}\text { Proteinuria } \\
\text { (gms/24 hrs) }\end{array}$ & $\begin{array}{c}\text { No. of cases } \\
\text { (n= 52) }\end{array}$ & Percentage \\
\hline 1. & $3.5-5.0$ & 14 & 26.9 \\
\hline 2. & $5.1-7.5$ & 17 & 32.7 \\
\hline 3. & $7.60-10.00$ & 12 & 23.1 \\
\hline 4. & $10.1-15$ & 5 & 9.6 \\
\hline 5. & More than 15 & 4 & 7.7 \\
\hline
\end{tabular}


Table III: Lipid profile in patients studied.

\begin{tabular}{|c|l|c|c|}
\hline S. No. & Lipid profile $(\mathbf{n}=\mathbf{5 2} ; \mathbf{m g} / \mathbf{d l})$ & Range & Mean S.D. \\
\hline 1. & Total Serum cholesterol & $238-462$ & $341.5 \pm 59.7$ \\
\hline 2. & Triglycerides & $184-355$ & $272.6 \pm 44.9$ \\
\hline 3. & High density lipoproteins (HDL) & $33-53$ & $42.9 \pm 3.3$ \\
\hline 4. & Low density lipoproteins (LDL) & $180-357$ & $244.9 \pm 53.0$ \\
\hline 5. & Very low density lipoproteins (VLDL) & $37-71$ & $54.6 \pm 8.9$ \\
\hline 6. & Phospholipids & $325-600$ & $434.6 \pm 80.5$ \\
\hline
\end{tabular}

Table IV: Incidence of primary glomerular disease as a cause of nephrotic syndrome in various studies.

\begin{tabular}{|l|c|c|}
\hline Pesce and First $^{7}$ & 1979 & $\mathbf{7 0 . 0 \%}$ \\
\hline Adu et al. $^{8}$ & 1981 & $86.0 \%$ \\
\hline Aggarwal $^{9}$ & 2000 & $58.5 \%$ \\
\hline Vikrant et al. $^{10}$ & 2004 & $84.8 \%$ \\
\hline Present study & 2006 & $76.9 \%$ \\
\hline
\end{tabular}

\section{Discussion}

The objective in patient management is to establish etiology and pathogenesis, which may lead to exact therapy. Renal diseases are often described in terms of clinical syndromes, recognized by symptoms, signs and laboratory tests available with little on no risk to patient. Syndrome analysis serves to narrow the number of potential causes and assist in selection of therapy, if available. Biopsy results, by narrowing further the number of potential causes and help establish pathogenesis, may aid in selecting appropriate therapy, or in avoiding inappropriate treatment which has a substantial risk. Nephrotic syndrome is one of the clinical expressions of renal glomerular disorder. Following the introduction of renal biopsy, it was found that many different histopathological appearances may underlie nephrotic syndrome, some of which cannot be guessed by clinical features. This makes needle biopsy of the kidney a central part of the evaluation, at least in adults. ${ }^{5,6}$

The diagnosis of primary glomerular disease was made in $40(76.9 \%)$ patients and that of secondary glomerular disease in $12(23.1 \%)$ cases. All the cases of primary glomerular disease were seronegative for Hepatitis B surface Antigen (HBsAg) and HIV, nor there was any other associated secondary cause present.

Incidence of primary glomerular disease as a cause of nephrotic syndrome in various studies are mentioned in Table IV. As is evident we obtained comparable data with respect to the other workers preceding, except Aggarwal ${ }^{9}$ who reporting primary glomerular disease in only $58.5 \%$ of his cases, derives this percentage from a patient subset with $20.14 \%$ having diabetic glomerular disease.
The mean value of proteinuria in our study was $7.15+$ $3.27 \mathrm{gms} / 24 \mathrm{hrs}$. It was consistent with the finding of Paone et al. ${ }^{11}$ In our study proteinuria ranged from 3.9-16.8 gms $/ 24 \mathrm{hrs}$. The heaviest proteinuria of $16.8 \mathrm{gms} / 24 \mathrm{hrs}$ was observed in a case of renal amyloidosis and 1 case of membranous glomerulonephritis had a proteinuria of $16.10 \mathrm{gms} / 24 \mathrm{hrs}$. Overall mean of proteinuria was highest in patients of renal amyloidosis, followed by patients of membranous glomerulonephritis. According to Glassock ${ }^{12}$, massive proteinuria is more likely to be associated with the glomerular disease of amyloidosis, membranous glomerulonephritis and focal segmental glomerulosclerosis (FSGS). The finding of heavy proteinuria in patients of membranous glomerulonephritis is also consistent with the finding of Falk et al ${ }^{13}$, but in our study in contrast to Glassock ${ }^{12}$, patients with FSGS as a group had comparatively less severe proteinuria and patients with membranoproliferative glomerulonephritis and minimal change disease had more proteinuria.

The overall level of blood urea and serum creatinine was raised in our study of patients with nephrotic syndrome. In 26 patients blood urea was more than $45 \mathrm{mg} / \mathrm{dl}$ and in 27 patients serum creatinine was more than $1.2 \mathrm{mg} /$ dl. Excluding chronic glomerulonephritis and diabetic nephropathy, the mean level of blood urea and serum creatinine was in the range of $42.8+8.7$ to $52.8+8.8 \mathrm{mg} /$ $\mathrm{dl}$ and $1.30+0.41$ to $1.80+0.76 \mathrm{mg} / \mathrm{dl}$ respectively, the mean level of blood urea was lowest in the group of patients with minimal change disease and highest in focal segmental glomerulosclerosis, whereas the mean level of serum creatinine was lowest in mesangioproliferative 
glomerulonephritis and highest in membranous glomerulonephritis. The degree of renal dysfunction could not be made out confidently by identifying these particular morphological groups. Similar view has been expressed by Park ${ }^{14}$ and Bohle et al ${ }^{15}$. The highest level of blood urea and serum creatinine was observed in patient with chronic glomerulonephritis. The high level in patient with chronic glomerulonephritis can be explained by the chronic and persistent nature of the renal damage.

Hyperlipidemia forms an important component of nephrotic syndrome. ${ }^{13,16}$ All the parameters of lipid profile were increased in most of the patients in our study, except for HDL, which was within normal range in majority of the patients. Total serum cholesterol was in the borderline high range in only three patients and in rest of the patients it was high. Triglycerides level was in borderline high category in 6 patients and in the rest it was high. LDL level was raised in all the patients studied. VLDL level was also raised in all the patients studied. Phospholipids were in the normal range in 11 patients and in the rest it was raised, the level being raised in those patients who had higher serum cholesterol level, whereas HDL levels was decreased in 6 patients. According to Alpers ${ }^{16}$, in patients of nephrotic syndrome total serum cholesterol, triglycerides, LDL and VLDL are raised in majority of patients, and few patients may have decreased HDL concentration.

Post biopsy histopathological diagnosis lead to change in therapy in $67.4 \%$ of the patients in our study. In the study of Cohen et al ${ }^{2}$, post biopsy diagnosis lead to change in therapeutic approach in $35 \%$ of patients of nephrotic syndrome. In the study of Paone and Meyer ${ }^{11}$, post biopsy histopathological diagnosis lead to therapeutic change in $33.3 \%$ of patients. However in the study of Richard ${ }^{17}$, they have documented that result of renal biopsy altered management in $86 \%$ of cases of nephrotic range proteinuria. Our observation of change in therapy was lower than that observed by Richard ${ }^{17}$, however it was higher than the observation of Cohen et al ${ }^{2} \&$ Paone and Meyer $^{11}$.

The alteration of therapy post biopsy in the present study most commonly revolved around the use of corticosteroid, and reflected both the identification of lesions perhaps responsive to certain type of therapy from those not responsive and vice versa. There was addition of corticosteroid in 1 patient, corticosteroid were discontinued in 11 patients, and increase in dose and duration of corticosteroid therapy in 15 patients. Similar views have been expressed by Cohen et al. ${ }^{2}$ In 8 patients post-biopsy, therapy with cytotoxic drug was started. Without biopsy these patients would not have had received the potentially beneficial treatment. Similar views have been expressed by Ponticelli et al. ${ }^{18}$ Changes in therapeutic intervention postbiopsy reflected that out of the 52 cases in our study, 21.2 $\%$ might have been overtreated. Cohen et $\mathrm{al}^{2}$ has expressed similar views that substantial number of patients may be overtreated, if the renal biopsy is not performed.

Thus to summarize, the estimate of prognosis was in agreement (pre-biopsy and post-biopsy) in $61.5 \%$ of cases whereas in $20(38.5 \%)$ patients it was different. In $12(23.1 \%)$ cases a better prognosis was estimated and in $8(15.4 \%)$ cases it was worse than estimated previously. Hence like Kark ${ }^{19}$ suggested in 1968, we also recommend that 'a renal biopsy study should be done to evaluate any case of diffuse renal disease in which there are major unsolved problems regarding etiology, in which there is a need to define prognosis, or to select or continue treatment by study of histologic changes in kidney, provided no contraindication exists'. These criteria still seem appropriate today.

\section{References}

1. Heptinstall RH. Pathology of the kidney. $3^{\text {rd }}$ ed. Vo. II. Boston/Toronto: Little Brown \& Co. 1983 pp 637-654

2. Cohen AH, Nast CC, Adler SG, Kopple JD. Clinical utility of kidney biopsies in the diagnosis and management disease. Am J Nephrology 1989;9:309-315.

3. Manaligod JR, Pirani CL. Renal biopsy in 1885. Semin Nephrol 1985;5:237-239.

4. Silkensen JR, Kasiske BL. Laboratory assessment of kidney disease: Clearance, urinalysis, and kidney biopsy. In, Brenner BM (ed.): Brenner and Rector's The Kidney. Seventh edn. (Vol. I) Saunders. An imprint of Elsevier. 2004: pp 1107-1150.

5. Cameron JS. The nephrotic syndrome and its complications. Am J Kidney Dis 1987;10:157-171.

6. Gulati S, Sharma AP, Sharma RK, Gupta A, Gupta RK. Do current recommendations for kidney biopsy in nephrotic syndrome need modifications? Pediatr Nephrol 2002; 17(6):404-408.

7. Pesce AJ, First MR. Proteinuria: an integrated review. New York: Marcel Dekker. 1979: pp 131.

8. Adu D et al. The nephrotic syndrome in Ghana: clinical and pathological aspect. Quart J Med 1981;50:297-306.

9. Aggarwal SK, Dash SC. Spectrum of renal diseases in Indian adults. J Assoc Physicians India 2000;48(6):594-600.

10. Vikrant S, Kaushal SK, Sharma A, Parashar BS. Spectrum of renal disease among admitted patients at a tertiary care hospital in Himachal Pradesh. Indian J Nephrol 2004;14:99195.

11. Paone DB, Meyer LRE: The effect of biopsy on therapy in renal disease. Arch Intern Med 1981;141:1039-1041. 
12. Glassock RJ. Proteinuria. In, Massry SG and Glassock RJ (eds.) Massry and Glsssock's Textbook of Nephrology. $4^{\text {th }}$ ed. Philadelphia: Lippincott William and Wilkins. 2001: pp 555.

13. Falk RJ, Jennette JC, Nachman PH. Primary glomerular disease. In: Brenner B.M. (ed.) Brenner and Rector's The Kidney, $7^{\text {th }}$ edn. Vol. 2. W.B. Saunders, Philadelphia. 2004; pp 1293-1380.

14. Park MH,D `Agathi V,Appel GB, Pirani CL. Tubulointerstitial disease in lupus nephritis. Relationship to immune deposits, interstitial inflammation, glomerular changes, renal function and prognosis. Nephron 1986;44:309-319.

15. Bohle A, Mackensen-Haen S, Gise H, et al. The consequences of tubulointerstitial; changes for renal function in glomerulopathies. In: Amerio A, Cortelli p, Massry SE (eds.) Tubulointerstitial nephropathies. Boston, Dordrecht, London: Kluwer.1991, pp 29-40.

16. Alpers CE. The Kidney. In Kumar V, Abbas AK and Fausto $\mathrm{N}$ (ed.): Robbins and Cotran Pathologic basis of disease. $7^{\text {th }}$ edn. Saunders. An imprint of Elsevier. 2004; pp. 955-1021.

17. Richards NT, Dabry S, Howie AJ, et al. Knowledge of renal histology alters patient management in over $40 \%$ of patients. Nephrol Dial Transpl 1994;9:1255-1259.

18. Ponticelli C, Mihatsch MJ, Imbasciati E. Renal biopsy: performance and interpretation. In, Davison AM, Cameron JS, Grünfeld JP et al. (eds.). Oxford textbook of clinical nephrology. Second edition. Vol. I. Oxford medical publications. 1998: pp 157-171.

19. Kark RM. Renal biopsy. J Am Med Ass 1968; 205: 220-226.

*Corresponding author:

Dr. Sufian Zaheer, Professor, Department of Pathology, Room number 409, Fourth floor, College building, VMMC and SJH, New Delhi, India Phone: +919808363738

Email: fianzaheer@gmail.com

Financial or other Competing Interests: None. 